\title{
Revisiting Multi-Channel Communication to Mitigate Interference and Link Dynamics in Wireless Sensor Networks
}

\author{
Antonio Gonga*, Olaf Landsiedel ${ }^{\dagger}$, Pablo Soldati ${ }^{\ddagger}$, Mikael Johansson* \\ *KTH - Royal Institute of Technology, Sweden, \{gonga, mikaelj\}@ee.kth.se \\ †Chalmers University of Technology, Sweden, \{olafl\}@ chalmers.se \\ ${ }^{\ddagger}$ Huawei Technologies Sweden AB, \{pablo.soldati\}@ huawei.com
}

\begin{abstract}
Multichannel communication has been proposed as alternative to adaptive (single-channel) routing protocols for mitigating the impact of interference and link dynamics in wireless sensor networks. While several studies have advocated features of both techniques (not without running up against contradicting arguments) a comprehensive study that aligns these results is still lacking. This paper aims at filling this gap. We present an experimental testbed setup used to perform extensive measurements for both single-channel and multichannel communication. We first analyze single-channel and multichannel communication over a single-hop in terms of packet reception ratio, maximum burst loss, temporal correlation of losses, and loss correlations across channels. Results show that multichannel communication with channel hopping significantly reduces link burstiness and packet loss correlation. For multi-hop networks, multi-channel communication and adaptive routing show similar end-to-end reliability in dense topologies, while multichannel communication can outperform adaptive routing in sparse networks with bursty links.
\end{abstract}

\section{INTRODUCTION}

Interference and link dynamics constitute great concerns for the stability and performance of wireless sensor network protocols. These phenomena normally manifest in link burstiness, i.e, prolonged periods of time where packet transmissions from a sender to a receiver are lost [1]. Such spikes of packet losses cause delays and instability in communication protocols with potentially severe consequences in, e.g., critical processes in industrial automation [2] and health care [3], [4].

Link dynamics and interference depend on the environment where a WSN is deployed. For example, wide-band interference may stem from moving machinery in factory environments but also from microwave ovens in home or office environments.Narrow-band interference is triggered from co-located wireless communication technologies operating in overlapping frequency bands, such as 802.11 or other WSN deployments. In addition, temporal dynamics such as people moving in offices or other factors, such as changing humidity or temperature, trigger link dynamics [6].

In this paper we address an ongoing debate on how to mitigate the impact of link dynamics on communication protocols, and attempt to reconcile the standpoints of two opposing views: (1) multichannel communication, and (2) adaptive routing. A number of papers show that frequency diversity, e.g. by employing channel hopping, increases the resilience to interference and link dynamics [6], [8], [9], while others argue that adaptive routing provides sufficient, or even superior, results [2]. This paper aims at resolving this contradiction. We believe that it is important for our community to understand the advantages and limitations of channel hopping and adaptive routing now. For example, techniques where consecutive packets are transmitted on different frequencies require tight time synchronization, which demand high accuracy oscillators in the WSN hardware and complex software implementations. This results in higher production and deployment costs for channel hopping based approaches. On the other hand, channel hopping is adopted by more and more standards, such as IEEE 802.15.4e [11], ISA100.11a [12], and WirelessHART [13]. Even if high-end hardware and software implementations for these standards are becoming commercially available, it is important to understand in which scenarios frequency-hopping provides benefits, and where adaptive routing is more suited.

Compared to the study in [2], we extend the analysis from packet reception ratio to additional metrics, such as maximum burst loss length, and packet loss correlation in time and frequency. For multi-hop networks, we focus on both reliability and delay. Also, we collect both single-channel and multi-channel traces, use more nodes in our evaluations, and let nodes send more packets (our nodes transmit 100 times more packets than those in [2]). Based on this wide, statistical evidence our paper makes three distinct research contributions:

1) First, we study the impact of multi-channel communication for point-to-point (single-hop) communication. We evaluate the performance in terms of maximum delay (burst length), and inter-frequency correlation of packet losses, and demonstrate that frequency diversity improves all these performance criteria. Hence, we conclude that frequency diversity is a good measure against link burstiness, especially for critical systems with tight deadlines and high reliability requirements.

2) Second, we show that significant improvements only occur on intermediate links. Hence, unless the deployment is very sparse, one can find a sufficiently many good links on every channel to provide end-to-end connectivity that is on par with the multi-channel solution, or even better, in terms of end-to-end delay. These paths 
would readily be found using standard routing protocols, such as CTP [14] or RPL [15]. So, while our basic observations, performance metrics and arguments are complementary to [2], our general conclusions agree.

3) Third, we reconcile the opposing results and show the reason for the apparent contradiction: In single-hop communication, or on fixed topologies where adaptive routing is not an option (such as preplanned WirelessHART deployments) frequency diversity can yield significant reliability improvements. However, when routing topologies can be adapted to link dynamics and interference, our results indicate that adaptive routing without channel hopping provides on par with reliability.

The remainder of this paper is structured as follows: Section II reviews the background and related work. Section III describes the experimental study for single-channel and multichannel scenarios. Section IV evaluates single-hop results in terms of packet reception ratio, maximum burst length, link burstiness: temporal correlation, and inter-frequency correlation. In Section V evaluates multi-hop scenarios for singlechannel and channel hopping in presence of routing. In Section VI discusses the results. In Section VII we make conclusions.

\section{BACKGROUND AND RELATED WORK}

Packet loss bursts are characterized by periods of time where a sender node cannot successfully transmit information to the intended receiver. Such bursts increase delays and energy consumption, and decrease network throughput and reliability. This is a particular challenge in systems with strong reliability and delay requirements, such as real-time monitoring and closed-loop control of processes with fast dynamics.

Each wireless channel has its own specific fading, i.e., link dynamics, and a different level and realization of interference. Hence, channel hopping where consectutive packets are transmitted on different frequencies can be useful for improving reliability [9], [10], [16], [17], resilience to interference [6], and on-time packet delivery [18]. However, the use of multichannel communications does not come for free. It trades simplicity offered by single channel communications by an increased implementation complexity and synchronization cost.

In [2] they argue that routing is sufficiently adaptive to deal with link dynamics and interference. For example, link estimators in today's protocol stacks commonly select good, long term stable links that show a very low degree of dynamics while offering good routing progress [19]. Furthermore, maintaining a rich neighbor table, allows the routing protocol to quickly adapt to sudden interference [14], [15].

In contrast to these papers, we neither introduce a new frequency-hopping based MAC nor a new routing protocol. Instead, we evaluate both possibilities along side each other and evaluate their potential for reliable and low-delay communication in the presence of link dynamics and interference. An important difference with [2], which discusses multi-channel protocols based on observed single-channel traces, is that we collect and analyze real channel-hopping measurements.

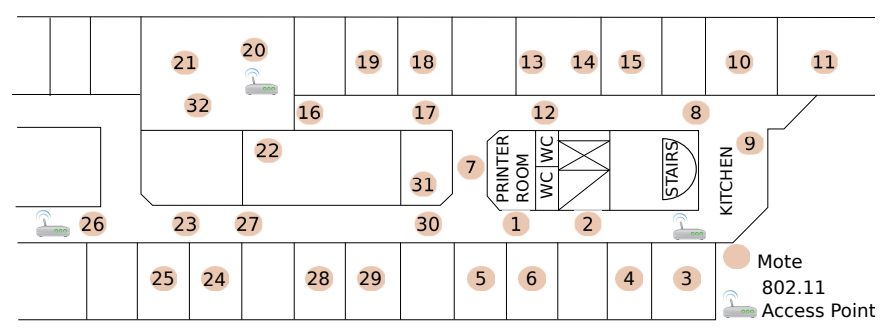

Fig. 1. Our testbed deployment consisting of 32 nodes. Three WiFi access points per floor, microwave ovens, and people moving in and in-between offices create different types of interference.

In a wider context, we have seen the combination of adaptive routing and slow hopping [7]; i.e., changing frequencies on the scale of hours, or when channel conditions deteriorate. Such approaches can be particularly useful for adapting to new sources of interference such as new network deployments.

\section{EXPERIMENTAL SETUP AND METHOdOLOGY}

To evaluate the benefits of both channel hopping and routing in mitigating link dynamics and interference, we collected an extensive set of experimental traces.

\section{A. Testbed}

Our testbed is located in our department at the third floor of a six story building. It consists of 32 TelosB motes [20] scattered throughout the ceiling of offices, corridors, and a kitchen area (see Fig. 1). Three 802.11 access points as well as other WSN deployments act as sources for narrow band interference. Additionally, microwave ovens, people moving throughout the day, and changes in temperature and humidity introduce wide-band interference and fading. We choose our local testbed over using one of the large, publicly available testbeds for two reasons: (1) Our evaluation bases on several days of experimental traces, which are difficult to collect in publicly available testbeds. (2) We used 32 nodes, leading to 992 links; this fact strikes a good balance between manageability of the data set and richness of data. Unlike the related work, e.g. [2], we do not study multi-channel RF characteristics on data collected on each separate channel, but we have a sevenfold larger set of data for channel-hooping sequences.

\section{B. Experimental Setup}

The data in our analysis is collected the following way: In round robin, each node transmits a burst of consecutive packets. Neighboring nodes log which of these packets they received. The burst length is 10,000 packets with an inter packet interval (IPI) of $10 \mathrm{~ms}$, since this is the time slot length used by standards such as WirelessHART. Scheduling nodes to transmit their burst after each other ensures that at our traces contain detailed short-term dynamics while at each point in time only a single node emits packets.

We collected traces for three scenarios: single channel, twochannel, and N-channel (see Table I).

- Single channel: In the single channel scenario, the experiment described above is conducted for each of the 
TABLE I

EXPERIMENT OVERVIEW: OUR ANALYSIS IS BASED ON THREE SETS OF EXPERIMENTS, LEADING TO A TOTAL OF MORE THAN 240 HOURS OF EXPERIMENTS AND 90 MILLION TRANSMISSIONS.

\begin{tabular}{|lrrrrc} 
Channels & Exp. & Nodes & Repeat & TX p.N. & Total TX \\
Single & 16 & 32 & 2 & 10,000 & $10,240,000$ \\
Two & 120 & 32 & 2 & 10,000 & $76,800,000$ \\
N & 5 & 32 & 2 & 10,000 & $3,200,000$
\end{tabular}

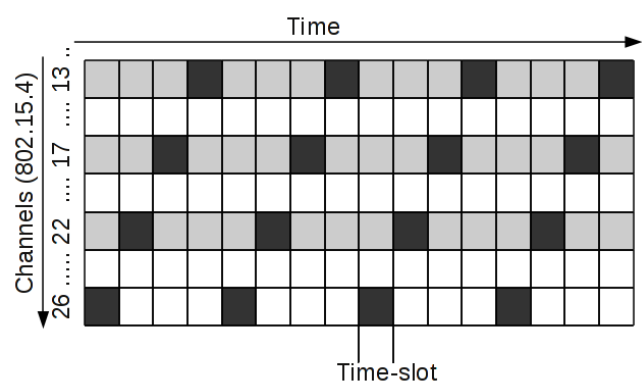

Fig. 2. An example of a network with a hopping sequence of 4 channels. Each consecutive packet (dark cells) is transmitted on a different channel. The rows in gray indicate channels interfered by the 802.11 standard.

16 channels in 802.15.4 standard. The goal of these experiments is to establish a baseline to which we compare the following multi-channel experiments.

- Two-channel: Each node alternates between two channels, transmitting packet $i$ on one frequency and packet $i+1$ on the other. This set is conducted for each possible combination of channels, leading to 120 experiments $(N \times(N-1) / 2$ for $N=16)$. Our goal is explore whether an increased gap between the two frequencies helps to decorrelates packet loss.

- N-channel: Based on the ISA 100 standard [12], we select hopping sequences of increasing length, i.e, 2, 4, 8, 12, and 16 channels (see Fig. 2). The sequences are selected to achieve a maximum gap between two consecutive frequencies (see Table II). As such large sequences are widespread in industry standards, it motivated us to explore where large sequences improve over short ones.

All experiments where conducted twice to detect and remove outliers. Overall, our three sets of experiments provide our analysis with a base of more than 240 hours of experiments and more than 90 Million transmissions (see Table I). We implemented our evaluation applications under the Contiki Operating System [21].

\section{SINGLE-Hop STUdY}

This section investigates the RF characteristic for pointto-point communication with single and multichannel communication. We compare single channel communication and channel hopping in four settings: (a) packet reception ratio (PRR), (b) maximum burst loss, (c) temporal correlation of losses (link burstiness), and (d) frequency correlation of losses.

\section{A. Packet Reception Ratio}

We begin this section studying how the packet reception ratio (PRR) varies across the 802.15.4 channels, consider-
TABLE II

Multi CHANNEL EXPERIMENTS: BASED ON INDUSTRY STANDARDS, WE SELECT HOPPING SEQUENCES WITH LARGE INTER-CHANNEL GAPS.

\begin{tabular}{l|rr}
$\# \#$ & Channel Sequence \\
2 & & 26,17 \\
4 & $26,19,12,20,24,22,17,13$ \\
8 & $26,19,12,20,24,16,23,18,25,22,17,13$ \\
12 & $24,16,23,18,25,14,21,11,15,22,17,13$
\end{tabular}

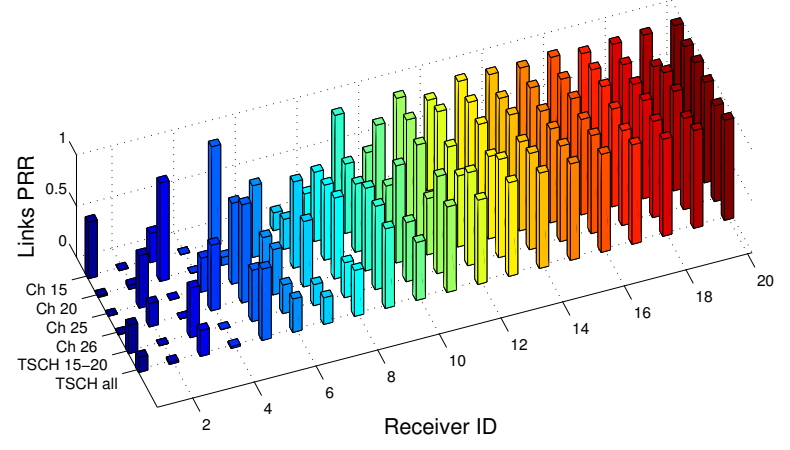

Fig. 3. Average links PRR for links in the network over the channels $c_{15}$, $c_{20}, c_{25}$ and $c_{26}$, and for the hopping sequences $S_{1}=\left\{c_{15}, c_{20}\right\}$ and $S_{1}=$ $\left\{c_{15}, c_{20}, c_{25}, c_{26}\right\}$.

ing four isolated channels (namely $c_{15}, c_{20}, c_{25}$ and $c_{26}$ ) and two TSCH sequences $S_{1}=\left\{c_{15}, c_{20}\right\}$ and $S_{2}=$ $\left\{c_{15}, c_{20}, c_{25}, c_{26}\right\}$.

We have selected a node in the testbed to transmit a burst of 10,000 packets sequentially on the chosen channels and TSCH sequences taken in the following order: $c_{15}, \quad c_{20}, \quad S_{1}, \quad c_{25}, \quad c_{26}, S_{2}$. We have run a hundred consecutive trials and the average PRR for links in the network is reported in Figure 3 for each channel and TSCH sequence. Note that this experiment different from the one described in Sec III, however, it combines scenarios of single-channel, twochannel hopping, and four-channels hopping scenarios. It was designed to compare PRR of TSCH and single channels by repeating a short experiment for 100 trials.

We obtained the average link PRR with TSCH sequences that reflects the average PRR of the link across the channels we used. Thus TSCH becomes useful when the goods channels are not known a priori, but it does not provide any gain when a link is reliable in all channels (e.g. for links 15-20). More interesting is the observation that about half of the links exist (or are good) only over some channels (i.e. PRR $>0$ ) but do not exist (or are very poor) over other channels $(P R R \approx 0)$. From the observed data, this behavior seems not to depend directly on WiFi interference. For instance, link 3 exists on channels $c_{15}$ and $c_{20}$ (subject to $\mathrm{WiFi}$ interference), but not in channels $c_{25}$ and $c_{26}$ (WiFi-free channels).

Our conclusion are aligned with the results in [2], [22] 


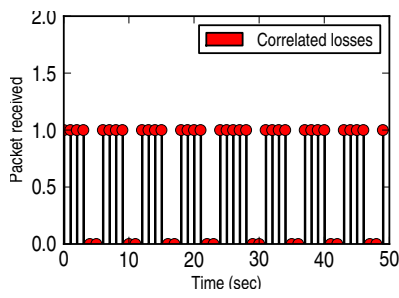

(a) Correlated losses.

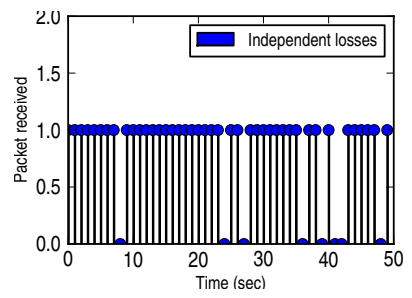

(b) Independent losses.
Fig. 4. An example of two links with two types of losses. Links with correlated receptions exhibit bursty behavior.

(i.e. the link quality is not homogenous across channels). However, the sole links PRR analysis, as used in [2], [22], is not sufficient to characterize the statistical behavior of a packet losses of a multi-channel channel hopping MAC. To close this gap, we next analyze three alternative metrics.

\section{B. Maximum Burst Loss}

Packet losses are often correlated in time and occur in bursts as illustrated in Figure 4. Therefore, analyzing only the (long term average) packet reception ratio is not sufficient, as it hides important performance indicator such as link burstiness. A simple metric to describe the link RF characteristics in terms of burstiness is the maximum burst loss, defined as the maximum number of consecutive packets lost over a communication link.

In what follows we analyze the maximum burst length for single- and multi-channel point-to-point communication for three scenarios:

- a WiFi-interference-free environment using channels $c_{25}$ and $c_{26}$ and the hopping sequence $S_{1}=\left\{c_{25}, c_{26}\right\}$;

- a WiFi-interfered environment using channels $c_{13}$ and $c_{22}$ and the hopping sequence $S_{2}=\left\{c_{13}, c_{22}\right\}$;

- and four longer hopping sequences defined in Table II.

For each case, we have collected data over the entire testbed as described in Section III, and we analyze the corresponding cumulative distribution function (CDF) of the experienced maximum burst length on all links in Figure 5.

Figures 5(a)-5(b) show the CDF of the maximum burst loss for Wi-Fi-free and Wi-Fi-interfered channels, respectively, for links with at least $90 \%$ PRR. In both scenarios, the individual channels are typically affected by longer bursts of losses compared to when the same channels are used with channel hopping. We further observe that the Wi-Fi-free channels $c_{25}$ and $c_{26}$ have experienced longer bursts than the Wi-Fi affected channels. It is also interesting to see that the behavior of the maximum burst loss with channel hopping is rather similar in both figures, i.e. is not affected by the Wi-Fi interference. This result is confirmed also in Figure 5(c) that shows that the $\mathrm{CDF}$ of the maximum burst loss does not benefit much from increasing the number of channels in the hopping sequence.

\section{Link Burstiness: Temporal Correlation}

To quantify the correlation of packet losses in time for single-channel communication, Srinivasan et al. defined a "link burstiness" metric referred to as the $\beta$-factor [1]. The metric is based on the conditional packet delivery function,
$C(n)$, which describes the conditional probability of successful packet reception given that the $n$ previous packet were received (for $n \geq 0$ ) or lost (for $n<0$ ). An "ideal bursty link" has $C(n)=1$ for $n>0, C(n)=0$ for $n<0$, and $C(0)=P R R$, while an independent link has $C(n)=P R R$ for all $n$. Now, the $\beta$-metric is defined as the KanatorovichWasserstein distance of the empirical link to an ideal bursty link, compared to the distance of an independent link with the same PRR. Specifically, the metric $\beta$ is defined as

$$
\beta=\frac{K W(I)-K W(E)}{K W(I)},
$$

where $K W(\cdot)$ is the distance from the ideal bursty link, while $E$ and $I$ are the conditional packet delivery functions of the empirical and independent link with the same PRR, respectively. A value of $\beta=0$ identifies a link with independent packet losses (i.e.following a Bernoulli process), while a value of $\beta=1$ indicates a bimodal link, i.e. a link that exists either in a good or a bad state. See [1] for more details.

We use $\beta$ to quantify the temporal correlation of packet losses for both single-channel and multi-channel communication. To compute $\beta$ in the case of channel hopping, we consider the sequence of packets received between each transmitterreceiver pair. Thus, in this case $\beta$ describes the link-burstiness of a given transmitter-receiver pair across multiple channels and for a given channel hopping sequence. Both the length of the hopping sequence and the channel used will influence $\beta$.

The results are illustrated in Figure 6. In particular, Figure 6(b) compares the CDF of $\beta$ for a data set collected with a length-4 channel hopping sequence $S=\left\{c_{26}, c_{22}, c_{17} c_{13}\right\}$, against the $\mathrm{CDF}$ of $\beta$ for data sets collected on each individual channel. The major insight from this result is that time synchronized channel hopping strongly reduces the packet loss correlation in time, with over $95 \%$ of links having a value of $\beta \leq 0.2$ corresponding to roughly independent packet losses. Packet losses on each individual channels are more correlated.

Figure 6(c) shows that using TSCH sequences with more channels (two to sixteen) can further reduce the packet loss correlation in time (since the same channel is sampled with a longer period). It is relevant to see that only a few channels are sufficient to obtain a relatively high decorrelation of the packet losses. For instance, with the length-2 hopping sequence (see Figure 6(a)) fewer than $10 \%$ of the links experienced high packet loss correlation $(0.5 \leq \beta \leq 1)$, while already with a length-4 sequence all links have $\beta<0.5$.

\section{Correlation of Losses: Frequency Correlation}

The same authors proposed another metric, referred to as $\kappa$-factor [23], to describe the inter-link reception correlation in case of single-channel communication. This metric was designed to quantify the spatial correlation of packet reception at different nodes receiving packets from the same source.

In our case, we revisit this metric to characterize the interfrequency reception correlation of a link in case of multichannel communication. Essentially, we adapt the theoretical framework of [23] to analyze the frequency-correlation of 


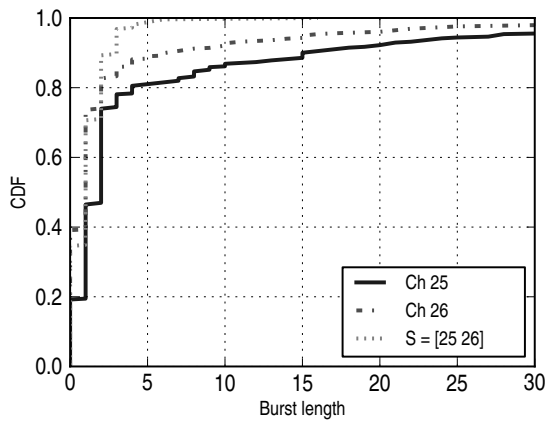

(a) Wifi-interference-free channels $c_{25}$ and $c_{26}$ and the hopping sequence $S_{1}=\left\{c_{25}, c_{26}\right\}$.

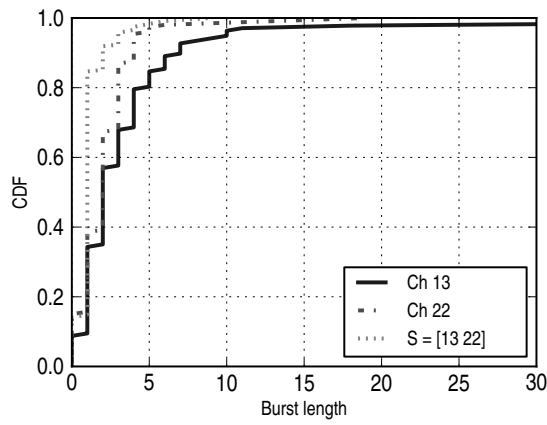

(b) Wifi-interfered channels $c_{13}$ and $c_{22}$ and the hopping sequence $S_{1}=\left\{c_{13}, c_{22}\right\}$.

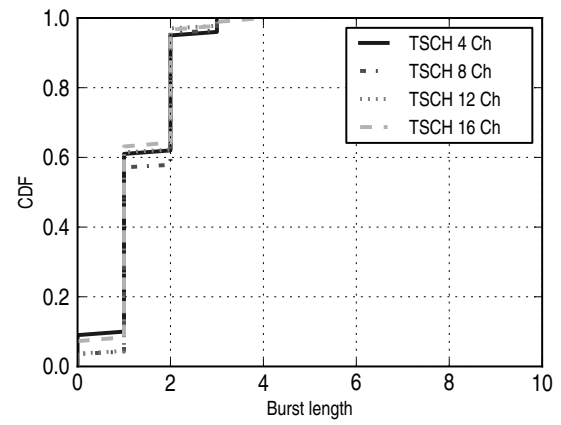

(c) Hopping sequences of length four to sixteen from Table III

Fig. 5. Cumulative distribution functions (CDFs) of the maximum burst length for single- and multi-channel communication for three relevant scenarios.

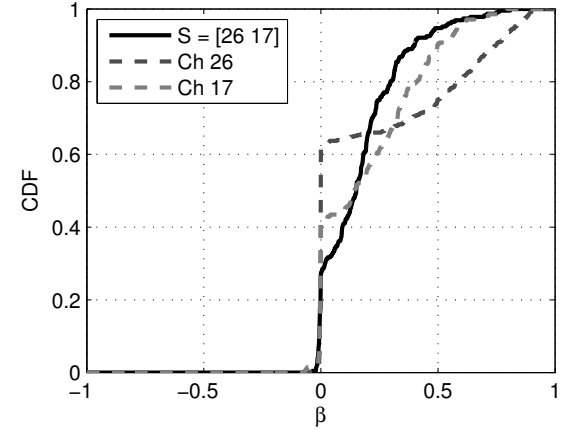

(a) Length 2 TSCH vs single-channels.

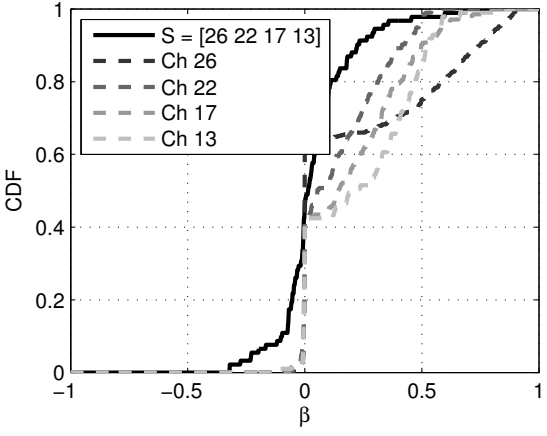

(b) Length $4 \mathrm{TSCH}$ vs single-channels.

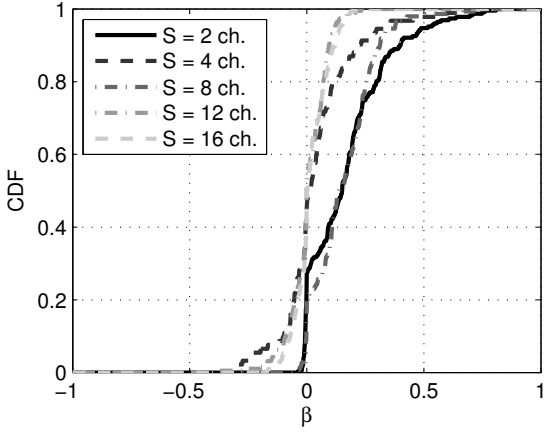

(c) TSCH lengths from 2 to 16.

Fig. 6. Study of the packet loss characteristics with single-channel and multi-channel point-to-point communication by the analysis of the $\beta-$ factor (time correlation).

packet reception in a link. For a given transmitter-receiver pair, let $x_{1}$ and $x_{2}$ denote two random variables representing 1 for a successful reception and 0 for a failure on channels $c_{1}$ and $c_{2}$, respectively. Following the steps in [23], we define define the correlation between the random variables $x_{1}$ and $x_{2}$ as

$$
\rho_{x_{1}, x_{2}}= \begin{cases}\frac{E\left[x_{1} \cdot x_{2}\right]-E\left[x_{1}\right] \cdot E\left[x_{2}\right]}{\sigma_{x_{1}} \cdot \sigma_{x_{2}}}, & \sigma_{x_{1}} \cdot \sigma_{x_{2}} \neq 0 \\ 0, & \text { otherwise }\end{cases}
$$

where $\sigma_{x}=\sqrt{E\left[(x-E[X])^{2}\right]}$ is the standard deviation of the variable $x ; E\left[x_{1} \cdot x_{2}\right]$ is the empirical mean of the product of $x_{1}$ and $x_{2}$, i.e the probability that a packet simultaneously transmitted on channels $c_{1}$ and $c_{2}$ is received correctly on both channels at the receiver; and $E\left[x_{1}\right]$ and $E\left[x_{2}\right]$ are the means of the random variables $x_{1}$ and $x_{2}$, i.e the packet reception ratio on channel $x_{1}$ and $x_{2}$ respectively. The inter-frequency reception correlation $\kappa$ can be defined as the normalized $\rho$,

$$
\kappa_{x_{1}, x_{2}}= \begin{cases}\frac{\rho_{x_{1}, x_{2}}}{\rho_{\max }}, & \text { if } \rho_{x_{1}, x_{2}}>0 \\ \frac{-\rho_{x_{1}, x_{2}}}{\rho^{\min }}, & \text { if } \rho_{x_{1}, x_{2}}<0 \\ 0, & \text { otherwise }\end{cases}
$$

where the quantities $\rho^{\min }$ and $\rho^{\max }$ are the minimum and maximum values of $\rho$ defined based on the packet reception ratio $P_{x_{1}}$ and $P_{x_{2}}$ for the two channels as

$$
\rho^{\min }=\frac{\min \left(P_{x_{1}}, P_{x_{2}}\right)-P_{x_{1}} \cdot P_{x_{2}}}{\sigma_{x_{1}} \cdot \sigma_{x_{2}}}
$$

and

$$
\rho^{\max }= \begin{cases}\frac{-P_{x_{1}} \cdot P_{x_{2}}}{\sigma_{x_{1}} \cdot \sigma_{x_{2}}}, & P_{x_{1}}+P_{x_{2}} \leq 1 \\ \frac{P_{x_{1}}+P_{x_{2}}-1-P_{x_{1}} \cdot P_{x_{2}}}{\sigma_{x_{1}} \cdot \sigma_{x_{2}}}, & \text { otherwise }\end{cases}
$$

In practice, the hardware limitation of the TelosB platform does not allow to send and/or receive the same packet simultaneously on two different channels. However, using channel hopping sequences consisting of two sole channels, and transmitting packets with IPI of $10 \mathrm{~ms}$, we have a fairly good approximation of the ideal behavior. Reducing the IPI may yield better approximation of the ideal case, but it is more demanding from a synchronization perspective.

In Figure 7 we analyze 2-channel hopping sequences in three settings: Figure 7(a) shows the CDF of $\kappa$ of three pairs of 802.15.4 adjacent channels affected by the interference of different WiFi channels and one pair of WiFi-free channels. The WiFi-free hopping sequence $S=\left\{c_{25}, c_{25}\right\}$ shows mild positive correlation for about $50 \%$ of the links in the network. The other hopping sequences are affected by different interference patterns (due to different Wi-Fi channels) which yield different patterns of correlations: packet losses on channels $c_{13}-c_{14}$ are uncorrelated over the network; less than $30 \%$ of the links have experienced channels $c_{19}-c_{18}$ weakly positive correlated packet losses; finally, about $50 \%$ of the links on channels $c_{22}-c_{24}$ have negative packet loss correlation. 


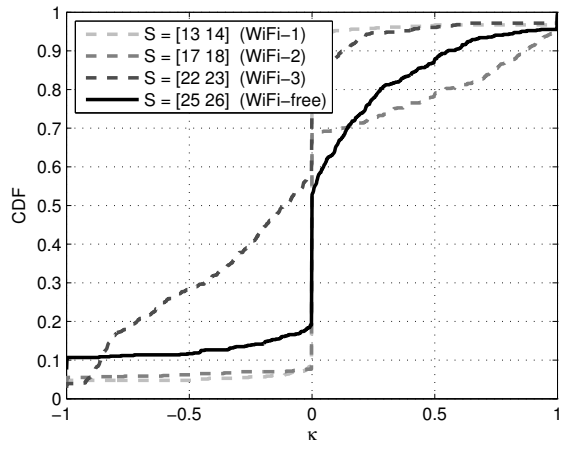

(a) Length-2 TSCH sequences with adjacent channels

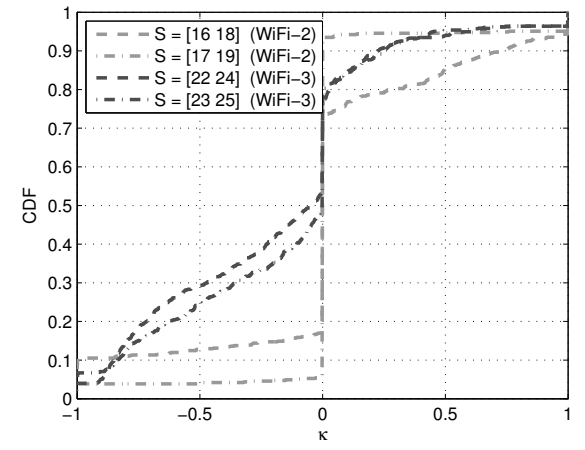

(b) Length-2 TSCH sequences with distant-2 channels

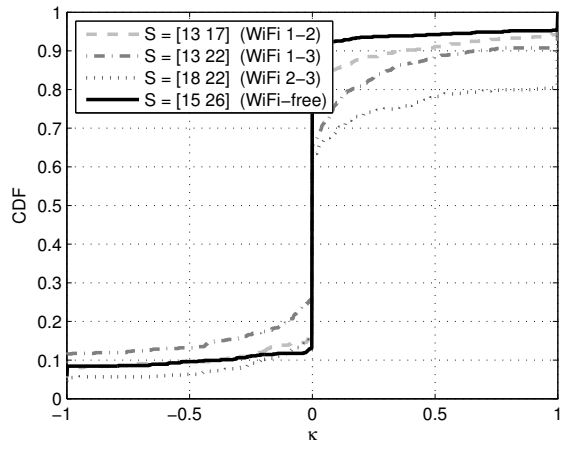

(c) Length-2 TSCH sequences with well separated channels

Fig. 7. Analysis of inter-frequency correlation through the CDF of the $\kappa$-factor for length-2 TSCH sequences computed over all links in the network. We consider three cases: (a) adjacent 802.15.4 channels under different 802.11 channels; (b) distant-2 802.15.4 channels under different 802.11 channels; and (c) well separated 802.15.4 channels.

Figure 7(b) shows a similar behavior for channel hopping sequences consisting of two non-adjacent channels subject to the same WiFi interference. Figure 7(c) shows that the packet loss correlation can be reduced by using channel hopping sequences with distant channels subject to different WiFi interference, but also with WiFi-free distant channels. It is worth noticing that using two well separated WiFi free channels over $85 \%$ of the links have experienced fully independent packet losses.

\section{Multi-Hop AnALysis}

In this section we evaluate the benefit of channel hopping in multi-hop single-path routing, and present two core findings: (1) We show that in dense and medium dense networks, channel hopping achieves similar end-to-end performance, such as average delay and reliability, as single-channel when the channels have roughly the same quality. The reason is that these networks have a sufficient number of long-term stable links with high PRR, for which the benefits of channel hopping are limited. Widespread routing metrics such as expected transmission count (ETX) [24] tend to find these links. For the same reason, when channels have different quality, channel hopping offers robustness by averaging out the behavior of the individual channels used. (2) In sparse topologies channel hopping can outperform single-channel communication.

\section{A. Routing: Channel Hopping in Multi-Hop Networks}

To evaluate the benefits of channel hopping for multi-hop routing, we build a routing tree [14], [15] on top of our experimental traces. We employ ETX [24] as routing metric, and select node 11 as source and node 26 as destination. These are the two nodes which are furthest away from each other in the network, allowing us to create routing paths with more hops (see Figure 1). For every transmitted packet from the sender, we allow each intermediate node to try to retransmit a packet a maximum of three times before discarding that packet. This value was chosen since the benefit of further retransmissions under multichannel communication are limited (cf. Figure 5(c)). We explicitly choose to base the evaluation on our traces (rather than an implementation) to avoid artifacts from the routing protocol and its implementation such as neighbor discovery or the link estimator employed. This ensures that our results are generic for the ETX routing metric and not bound to a specific protocol implementation.

In this setting, we explore the impact of network density on end-to-end reliability and delay. To create networks with varying density, we eliminate links from the experimental traces. Starting from the best link in terms of PRR, we remove links until the network becomes disconnected. We evaluate two key scenarios:

- WiFi interfered channels: We use two worst channels ${ }^{1}$ in our experiments and their hopping combination, namely channels $c_{13}$ and $c_{22}$. These channels are well separated in the spectrum and interfered by different 802.11 channels, thus fairly independent as showed in Figure 7(c).

- WiFi-free channels We use two less interfered channels and their channel hopping sequence, namely channels $c_{15}$ and $c_{26}$. These channels happen to have the best quality and are well separated in the frequency domain, thus well independent on a per-link basis.

Figure 8 compares the multi-hop end-to-end delay and reliability between the two strongly interfered channels and their channel hopping combination. Routing on top of channel hopping yields an average end-to-end delay that is essentially the average of the delay experience when routing on each individual channel, see Figure 8(a). On the other hand, channel hopping helps to reduce the maximum end-to-end delay and increases reliability, see Fig. 8(c) and Fig. 8(b) respectively, while the average hop-count is in line with the hop-count obtained with the worst channel, see Figure 8(d). Figures 9(a)9(d) show the same metrics for the WiFi-free channels pair $c_{15}$ and $c_{26}$. In this case channel hopping tends to achieve similar performance as the best channel.

The insight from these results is that when routing topologies can be adapted to link dynamics and interference, adaptive

\footnotetext{
${ }^{1}$ We select the worst and best channels in the network by analyzing the corresponding network-wide CDF of the links PRR.
} 


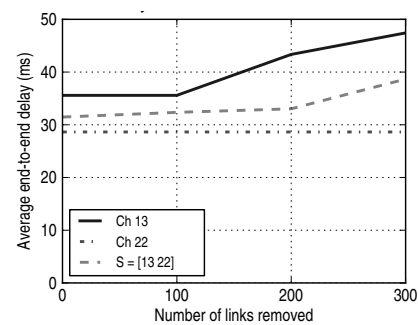

(a) Average end-to-end delay.

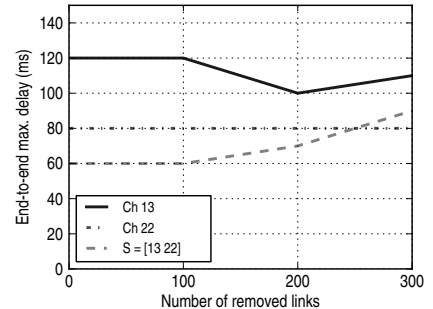

(b) Maximum end-to-end delay.

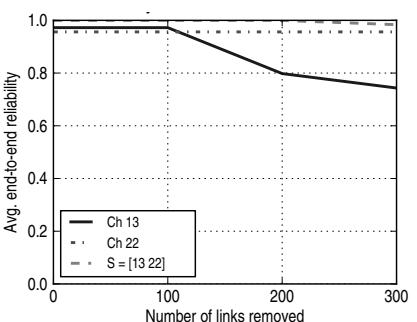

(c) Average end-to-end reliability.

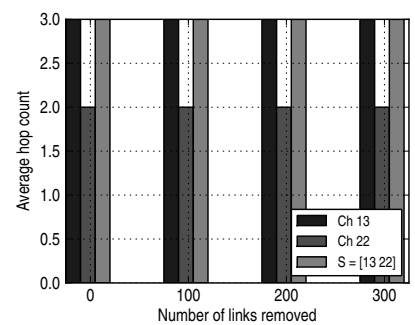

(d) Average hop count

Fig. 8. The Figure shows the end-to-end delay and reliability between two channels and their channel hopping combination. Single channel and multichannel hopping combination have roughly the same end-to-end delay while achieving lower delay spikes and increased reliability.

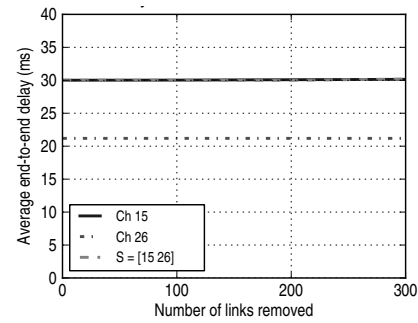

(a) Average end-to-end delay.

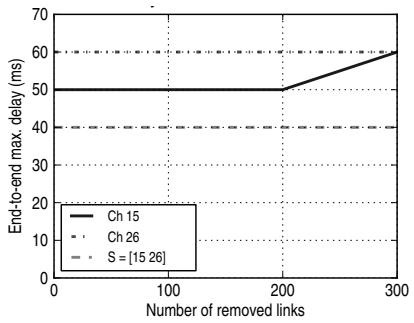

(b) Maximum end-to-end delay.

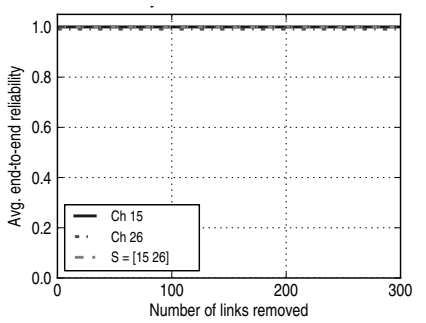

(c) Average end-to-end reliability.

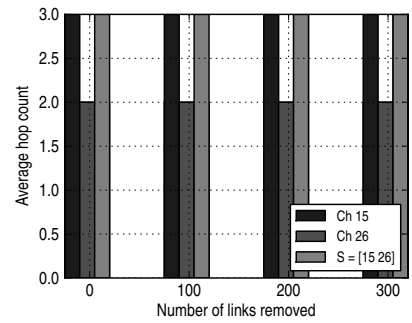

(d) Average hop count.

Fig. 9. This Figure shows that increasing channel robustness does not guarantee a better network performance compared to single channel solution. As long as there still exist many good links in the network, routing makes the use of channel hopping superfluous .

routing without channel hopping provides similar reliability as multichannel communication. This can be explained by observing that adaptive routing protocols rely on link estimators that commonly select good, long-term stable links. These links show a very low degree of dynamics while offering good routing progress [19]. On the other hand, the frequency diversity offered by multi-channel communication helps reduce the average end-to-end delay and the maximum delay in the network. To achieve the same with a adaptive routing on a single channel one would need to know a priori which channel experiences the good conditions network-wide.

\section{B. Multi-Hop Analysis in Sparse Networks}

In sparse network topologies, however, channel hopping can outperform adaptive routing. To analyze this scenario we use use channels $c_{13}$ and $c_{22}$ and their hopping sequence on the network topology depicted in Figure 10(a). This scenario, where routing has less flexibility and links have intermediate PRR, is a typical representative of standard protocols such as WirelessHART where routing changes occur at very low rate to avoid excessive overhead from computing and disseminating link schedules.

Figures 10(b) and 10(c) compare the average end-to-end delay and reliability of various end-to-end paths using singlechannel and channel hopping communication. Due to the limited path diversity and the intermediate quality of links, multichannel communication reduces end-to-end delay and increases reliability with gains ranging from $15 \%$ to $90 \%$ when compared to single channel communication.

\section{DISCUSSION}

Our paper is an attempt to align the opposite standpoints on whether multichannel communication or adaptive routing is the better solution for mitigating the impact of link dynamics on communication protocols. Section IV illustrates that the link PRR across individual channels is not uniform, while channel hopping tend to achieve the average PRR on the selected channels. Thus, papers arguing that multichannel communication can improve reliability on a single link are neither wrong or right a priori, i.e. it depends on the ability to select the good channel for communication.

On the other hand, the sole PRR is not sufficient characterize the packet loss correlation and link burstiness, which represents the main challenge in systems with strong reliability and delay requirements, such as real-time monitoring and closed-loop control of processes with fast dynamics. For these applications, we have used other metrics to show frequency diversity is a good measure against link burstiness as it decorrelates packet losses both across time and frequency domain, thus reducing the number of consecutive packet losses compared to the corresponding individual channels. Therefore, in single-hop communication, or on fixed topologies where adaptive routing is not an option (such as preplanned WirelessHART deployments) frequency diversity can yield significant reliability improvements.

However, when routing topologies can be adapted to link dynamics and interference, our results indicate that adaptive routing without channel hopping provides on par with reliability. This can be explained by observing that significant reliability improvements only occur on intermediate links. These links, however, are rarely used for routing as link estimators in today's protocol stacks commonly select good, long term stable links that show a very low degree of dynamics while offering good routing progress [19]. Hence, unless the deployment is very sparse, one can find a sufficiently many 


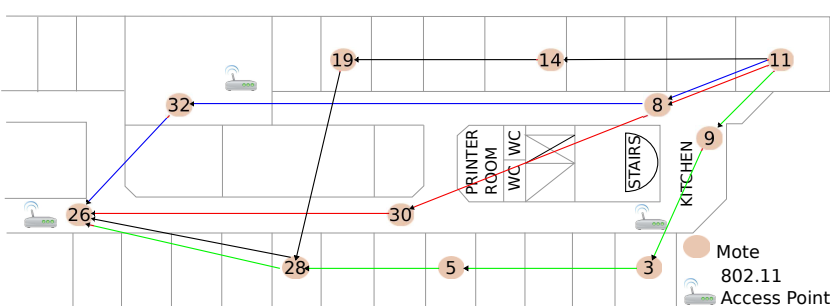

(a) An example of a sparse network topology.

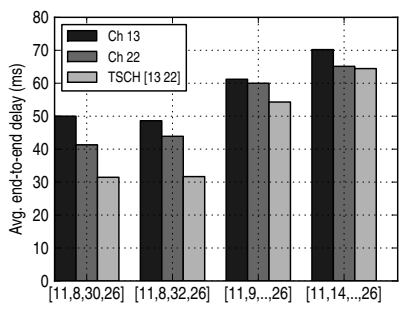

(b) Average end-to-end delay

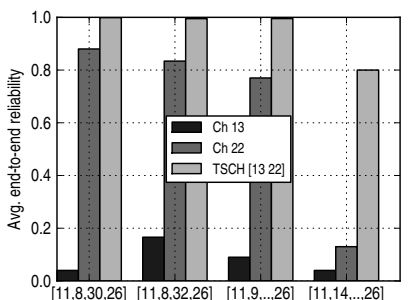

(c) Average end-to-end reliability

Fig. 10. comparison between single-channel and TSCH in a sparse network. Channel hopping outperforms single-channel in both the average end-to-end delay, and reliability.

good links on every channel to provide end-to-end connectivity that is on par with the multi-channel solution, or even better, in terms of end-to-end delay. These paths would readily be found using standard routing protocols, such as CTP [14] or RPL [15]. So, while our basic observations, performance metrics and arguments are complementary to [2], our general conclusions agree.

\section{CONCLUSION}

This paper addresses the ongoing debate on whether multichannel communication or adaptive routing is more suitable to mitigate the impact of link dynamics in wireless sensor network. To align different views on this topic, we conducted an extensive measurement campaign in our testbed, collecting over 90 Millions of data packets for both single-channel and multichannel communication.

Based on this data, this paper analyses single-channel and multi-channel communication over a single-hop in terms of packet reception ratio, maximum burst loss, temporal correlation of losses, and frequency correlation of losses. Our results show that, on a single-hop, multichannel communication via channel hopping significantly reduces link burstiness and decorrelates packet losses, both in time and frequency, to the point that $95 \%$ of links show independent packet losses for hopping sequences with more than two channels.

In multi-hop networks, multi-channel communication and adaptive routing yield similar end-to-end reliability in dense and medium dense deployments. This can be explained since routing protocols tend to use good long-term stable links, thus avoiding the intermediate links where the burstiness behavior is more dominant. In sparse networks, where adaptive routing looses its flexibility, multichannel yields better performance in terms of both average end-to-end delay and reliability.

\section{REFERENCES}

[1] K. Srinivasan, M. A. Kazandjieva, S. Agarwal, and P. Levis, "The $\beta$ Factor: Measuring Wireless Link Burtiness," Sensys 08, 2008.

[2] J. Ortiz and D. Culler, "Multichannel Reliability Assessment in Real World WSNs," IPSN10, April 12-16, 2010, Stockholm, Sweden, 2010.

[3] J. Ko, C. Lu, M. B. Srivastava, J. A. Stankovic, A. Terzis, and M. Welsh, "Wireless Sensor Networks for Healthcare," In Proceedings of the IEEE, 2010.

[4] O. Chipara, C. Lu, T. C. Bailey, and G.-C. Roman, "Reliable Clinical Monitoring using Wireless Sensor Networks: Experiences in a Stepdown Hospital Unit," in Proceedings of ACM Sensys'10, November 3-5, 2010, Zurich, Switzerland, 2010.
[5] C. A. Boano, T. Voigt, C. Noda, K. Romer, and M. Zuniga, "JamLab: Augmenting sensornet testbeds with realistic and controlled interference generation," IPSN'11, April 12-14, 2011, Chicago , IL, 2011.

[6] T. Watteyne, S. Lanzisera, A. Mehta, and K. S. J. Pister, "Mitigating Multipath Fading Through Channel Hopping in Wireless Sensor Networks," IEEE International Conference on Communications (ICC), 2010, Cape Town, South Africa, 2010.

[7] M. Sha, G. Hackmann, and C. Lu, "ARCH. Practical Channel Hopping for Reliable Home-Area Sensor Networks," Real-Time and Embedded Technology and Applications Symposium (RTAS), 2011, 2011.

[8] T. Watteyne, A. Mehta, and K. Pister, "Reliability Through Frequency Diversity: Why Channel Hopping Makes Sense," in PE-WASUN, 2009.

[9] G. Zhou, C. Huang, T. Yan, T. He, and J. A. Stankovic, "MMSN: Multi-Frequency Media Access Control for Wireless Sensor Networks," INFOCOM 2006, pp. 1 - 13, 2006.

[10] Y. Wu, J. A. Stankovic, T. He, J. Lu, and S. Lin, "Realistic and Efficient Multi-channel Communications in Wireless Sensor Networks," INFOCOM'08, pp. 1193 - 1201, 2008.

[11] IEEE802154e, Last Access January 2012. [Online]. Available: http: //ieee802.org/15/pub/TG4e.html

[12] ISA10011a, Last Access January 2012. [Online]. Available: http://www. isa.org//MSTemplate.cfm?MicrositeID=1134\&CommitteeID=6891

[13] WirelessHART, Last Access January 2012. [Online]. Available: http://www.hartcomm.org/hcf/documents/documents_specifications.html

[14] O. Gnawali, R. Fonseca, K. Jamienson, D. Moss, and P. Levis, "Collection Tree Protocol," in Proceedings of ACM Sensys'09, November 4-6, 2009, Berkeley, CA, USA, 2009.

[15] R. D. 19, Last Access January 2012. [Online]. Available: http: //tools.ietf.org/html/draft-ietf-roll-rpl-19

[16] W.-Z. Song, R. Huang, B. Shirazi, and R. LaHusen, "TreeMAC: Localized TDMA MAC Protocol for Real-Time High-data-rate Sensor Networks," PerCom 2009.

[17] Y. Kim, H. Shin, and H. Cha, "Y-MAC: An Energy-efficient Multichannel MAC Protocol for Dense Wireless Sensor Networks," IPSN, 2008.

[18] K. S. J. Pister and L. Doherty, "TSMP: Time Synchronized Mesh Protocol," in Proceedings of the IASTED International Symposium on Distributed Sensor Networks, 2008.

[19] R. Fonseca, O. Gnawali, K. Jamieson, and P. Levis, "Four-Bit Wireless Link Estimation," In Proceedings of the Sixth Workshop on Hot Topics in Networks (HotNets VI), 2007, 2007.

[20] TelosB, Last Access January 2012. [Online]. Available: http://bullseye.xbow.com:81/Products/Product_pdf_files/Wireless_ pdf/TelosB_Datasheet.pdf

[21] A. Dunkels, B. Gronvall, and T. Voigt, "Contiki - A Lightweight and Flexible Operating System for Tiny Networked Sensors," in Proceedings of IEEE LCN'04, 2004

[22] D. Sexton, M. Hahony, and M. Lapinski, "Radio Channel Quality in Industrial Wireless Sensor Networks," SICON'05, 2005.

[23] K. Srinivasan, M. Jain, J. I. Choi, T. Azim, E. Kim, P. Levis, and B. Krishnamachari, "The $\kappa$ Factor: Inferring Protocol Performance Using Inter-link Reception Correlation," MOBICOM 10, 2010.

[24] D. S. J. D. Couto, D. Aguayo, J. Bicket, and R. Morris, "A highthroughput path metric for multi-hop wireless routing," In Proceedings of the 9th annual international conference on Mobile computing and networking, 2003. 